\title{
The Fab-7 element of the bithorax complex attenuates enhancer-promoter interactions in the Drosophila embryo
}

\author{
Jumin Zhou, ${ }^{1}$ Scott Barolo, ${ }^{2}$ Paul Szymanski, ${ }^{3}$ and Michael Levine ${ }^{1,4}$ \\ ${ }^{1}$ Department of Molecular and Cellular Biology, Division of Genetics, University of California, Berkeley, California \\ 94720-3201 USA; ${ }^{2}$ Department of Biology, University of California at San Diego, La Jolla, California 92093 USA; \\ ${ }^{3}$ Amplicon, Inc., East Setauket, New York 11733 USA
}

Enhancers integrate positive and negative regulatory information to direct localized patterns of gene expression in the Drosophila embryo. Here we present evidence for the occurrence of cis regulatory elements that control the levels of gene expression by modulating enhancer-promoter interactions. For this purpose we have investigated the Drosophila bithorax complex (BX-C) because genetic studies suggest that the BX-C contains as much as $300 \mathrm{~kb}$ of cis regulatory information. A specialized DNA element, Fab-7, has been proposed to function as a boundary element that separates the $i a b-6$ and $i a b-7$ cis regulatory regions within the Abd-B domain of the BX-C. A 1.2-kb Fab-7 DNA fragment was placed between divergently transcribed white and lac $Z$ test promoters and challenged with several defined enhancers expressed in the early embryo. These studies suggest that Fab-7 functions as an attenuator, which weakens gene expression by reducing enhancer-promoter interactions. Fab-7 selectively blocks distal enhancers in an orientation-independent fashion, and can function when located far from either the distal enhancer or target promoter. Fab-7 may be related to insulator DNAs, which flank genetic loci and functionally isolate neighboring genes. We propose that specialized DNA elements, such as the Fab-7 attenuator, might play a general role in controlling the levels of gene expression by modulating enhancer-promoter interactions.

[Key Words: Drosophila; Fab-7; enhancer; attenuator]

Received August 30, 1996; revised version accepted October 29, 1996.

The Drosophila bithorax complex $(\mathrm{BX}-\mathrm{C})$ contains three Hox-containing transcription units, which encode homeotic selector proteins that control the patterning of thoracic and abdominal body segments (Lewis 1978; Sanchez-Herrero et al. 1985; Lewis et al. 1995; Martin et al. 1995). One of these, Abdominal-B (Abd-B), controls the morphogenesis of posterior regions, including parasegments (PS) 10 through 14 (Karch et al. 1985; Tiong et al. 1985; Casanova et al. 1986). The gene is regulated by a series of complex cis regulatory regions, iab-5 through iab-9, that restrict expression within specific parasegments (Karch et al. 1985; Celniker et al. 1989, 1990; DeLorenzi and Bienz 1990; Boulet et al. 1991; SanchezHerrero 1991; see Fig. 1). For example, the iab-6 region is thought to contain a series of enhancers that mediate expression within different tissues of PS 11 (e.g., Vazquez et al. 1993).

Transcriptional repression is essential for the establishment and maintenance of parasegment-specific patterns of $A b d-B$ expression. Previous studies have identified a 1-kb enhancer, IAB5, that directs expression

${ }^{4}$ Corresponding author. within PS 10, PS 12, and PS 14 (Busturia and Bienz 1993). Repression in anterior regions, PS 1 through 9, appears to be initiated by the gap proteins hunchback (hb), Kruppel $(\mathrm{Kr})$, and knirps (kni). This repression is thought to be maintained in older embryos (and larvae) by proteins encoded by the Polycomb group (PC-G; Wedeen et al. 1986; Busturia and Bienz 1993; Orlando and Paro 1993; Chan et al. 1994; Chiang et al. 1995; Zink and Paro 1995).

It has been proposed that boundary elements separate neighboring cis regulatory regions within the $A b d-B$ domain (Gyurkovics et al. 1990; Galloni et al. 1993; Karch et al. 1994). These may function in a manner analogous to insulators, which functionally isolate neighboring genes by blocking interactions of cis elements with inappropriate target promoters (Kellum and Schedl 1991 1992; Geyer and Corces 1992; Dorsett 1993). Previous studies have identified two potential boundary elements, Mcp and Fab-7 (Gyurkovics et al. 1990; Galloni et al. 1993; Karch et al. 1994). Mcp resides between iab-4 and $i a b-5$, whereas Fab-7 maps between $i a b-6$ and $i a b-7$ (summarized in Fig. 1). Mutations in Fab-7 result in the misregulation of $A b d-B$ in PS 11 , and a corresponding homeotic transformation of PS 11 tissues (sixth abdominal segment; A6) into PS 12 (A7; Gyurkovics et al. 1990; Galloni et al. 1993). $A b d-B$ is normally expressed in an 


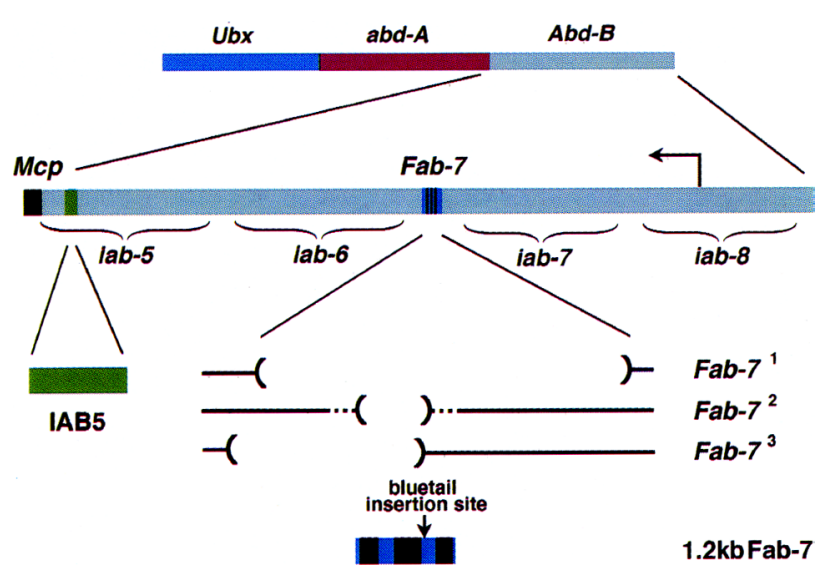

Figure 1. Summary of the BX-C. The complex contains three Hox transcription units, Ultrabithorax (Ubx), abdominal-A $|a b d-A|$, and $A b d o m i n a l-B(A b d-B)$. The cis regulatory regions associated with $A b d-B$ are shown below. The leftward arrow indicates the position of the $A b d-B$ transcription start site. The $1-\mathrm{kb}$ IAB5 enhancer is located in a distal region of $i a b-5,57 \mathrm{~kb}$ downstream of the $A b d-B$ transcription start site. The Fab-7 element is located between $i a b-6$ and $i a b-7$. A blow-up of the element is shown below. It contains three DNaseI-hypersensitive sites (indicated by shading). The blue horizontal bars indicate regions that are deleted in different Fab-7 mutations. For example, the Fab- $7^{2}$ allele deletes two of the three hypersensitive sites (Karch et al. 1994). The 1.2-kb Fab-7 fragment used in this study (bottom-most bar) contains all three hypersensitive sites.

anteroposterior gradient, with highest levels in posterior regions (PS 12, PS 13, and PS 14), and progressively lower levels in PS 11 and PS 10 (Harding and Levine 1988; Kuziora and McGinnis 1988; Celniker et al. 1989; DeLorenzi and Bienz 1990; Boulet et al. 1991). Fab-7 mutants display similar levels of $A b d-B$ expression in PS 11 and PS 12 (Galloni et al. 1993).

Previous studies led to several proposals regarding the nature of Fab-7. Perhaps removal of Fab-7 results in the ectopic activation of $i a b-7$ enhancers in PS 11 where they are normally silenced by Pc-G repressors (Vazquez et al. 1993). According to this view, Fab-7 separates iab-6 and iab-7 into distinct chromatin (or loop) domains. Alternatively, it has been suggested that Fab-7 functions as a silencer element, which attenuates $A b d-B$ expression in anterior regions, including PS 11 (Busturia and Bienz 1993). Evidence that Fab-7 is a boundary element is provided by the observation that a transgene inserted between Fab-7 and iab-7 is activated by iab-7 enhancers, but not by distal $i a b-5$ or $i a b-6$ regulatory elements, including the IAB5 enhancer (Galloni et al. 1993; see Fig. 1). In contrast, the notion that Fab-7 functions as a silencer, or Polycomb response element (PRE), is suggested by the analysis of transgenic fly strains containing a large (3.7-kb) Fab-7 DNA fragment attached to various heterologous enhancers and promoters. Fab-7 was shown to silence a mini-white gene and lacZ reporter; this silencer activity correlated with the binding of Pc protein to the integrated transgenes (Zink and Paro 1995). Fab-7 also silences the anterior expression of a $b x d-U b x$ fusion promoter that is normally expressed throughout most of the embryonic CNS; this repression is lost in PC mutants (Busturia and Bienz 1993).

In the present study we analyzed the activities of a 1.2-kb Fab-7 DNA fragment in the context of synthetic gene complexes containing defined enhancers expressed in the precellular embryo. We show that this Fab-7 fragment selectively blocks or attenuates distal, but not proximal, enhancers. A variety of enhancers were examined, including IAB5, the even-skipped (eve) stripe 3 enhancer (Small et al. 1996; Yan et al. 1996), the hairy stripe 1 enhancer (Howard and Struhl 1990; Riddihough and Ish-Horowicz 1991) and the rhomboid lateral stripe enhancer (Ip et al. 1992). The latter enhancers were selected to determine whether Fab-7 functions as a spatially localized silencer, or parasegment-specific PRE. Our findings suggest that Fab-7 functions as an attenuator, possibly in a manner analogous to previously characterized insulator DNAs. We discuss the role of specialized DNA elements, such as attenuators, in gene regulation.

\section{Results}

A 1.2-kb Fab-7 fragment contains all three major DNaseI hypersensitive sites identified in previous studies (Karch et al. 1994; summarized in Fig. 1). It resides between the IAB5 enhancer and $A b d-B$ transcription unit (Busturia and Bienz 1993). As shown previously, the IAB5 enhancer directs a broad band of expression in parasegments (PS) 10 through 14 of transgenic, precellular embryos (Fig. 2D). This pattern is resolved into three stripes (PS 10, PS 12, and PS 14) after cellularization (Busturia and Bienz 1993).

\section{Fab-7 attenuates the IAB5 enhancer}

A fusion promoter containing the IAB5 enhancer and a heterologous head stripe enhancer from the hairy promoter region (H1; Howard and Struhl 1990; Riddihough and Ish-Horowicz 1991) directs an additive pattern of expression when placed between divergently transcribed white and lacZ reporter genes (Fig. 2A,C; see maps below the panels). Altered patterns of expression are observed when the neutral spacer sequence that separates the two enhancers is replaced by the Fab-7 element (Fig. 2B,D). The leftward white gene is expressed within the limits of the head stripe, whereas expression in the presumptive abdomen is nearly lost (cf. Fig. 2B and A). The opposite situation is observed for the rightward lac $Z$ reporter gene. In this case, expression is lost in the head, but normal staining is observed in the abdomen (cf. Fig. 2D and C).

These results suggest that Fab-7 selectively blocks the interactions of distal enhancers. This block appears to be equally effective whether the $5^{\prime}-3^{\prime}$ orientation of the Fab-7 element is the same as the target gene (Fig. 2D) or is inverted (Fig. 2B). Fab-7 does not appear to block all distal enhancers to the same extent. For example, it at- 

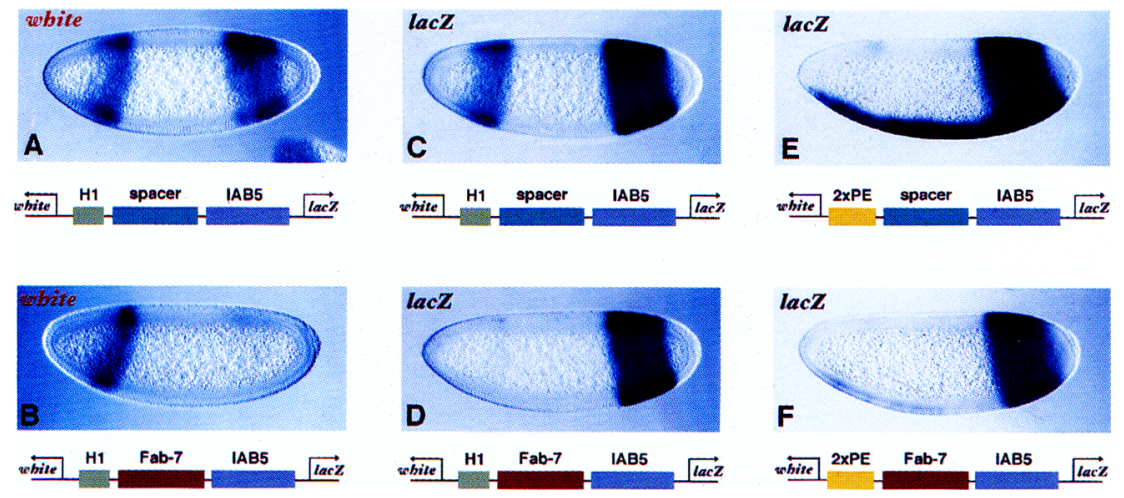

Figure 2. Fab-7 attenuates IAB5. Transgenic embryos were hybridized with either a digoxygenin-labeled white or lac $Z$ antisense RNA probe. Hybridization signals were visualized by histochemical staining using alkaline phosphatase. All embryos are undergoing cellularization, about $150 \mathrm{~min}$ postfertilization; they are oriented with anterior to the left and dorsal up. $(A \mid$ white expression pattern directed by the fusion promoter summarized in the diagram below the embryo. The synthetic gene complex contains two divergently transcribed genes, the leftward white gene and a rightward $l a c Z$ reporter gene /driven by a minimal eve promoter se-quence). Two different enhancers were placed between the white and lac $Z$ transcription units; a 200-bp enhancer that directs expression of hairy stripe $1(\mathrm{H} 1)$ as well as the $1-\mathrm{kb}$ IAB5 enhancer from the $i a b-5$ cis regulatory region of $A b d-B$. The two enhancers are separated by a $1-\mathrm{kb}$ spacer sequence. In this experiment, the spacer was obtained from a matrix attachment region (MAR) of the mouse $\mu$ light chain gene. An additive white expression pattern is observed consisting of a head stripe (directed by the H1 enhancer) and a band of staining in the presumptive abdomen (directed by IAB5). $(B)$ Same as $A$ except that the spacer sequence was replaced with the 1.2-kb Fab-7 element. The distal IAB5 enhancer is attenuated, so that there is only weak expression of the white reporter gene in the abdomen. In contrast, the proximal $\mathrm{Hl}$ enhancer is unaffected and directs a strong head stripe. $(C)$ Expression of the rightward $l a c Z$ gene directed by the same fusion promoter shown in $A$. As for white, the fusion promoter directs an additive staining pattern consisting of a head stripe and abdominal band. $(D)$ Same as $C$ except that the spacer sequence was replaced with the Fab-7 element. The distal $\mathrm{Hl}$ enhancer is selectively blocked, so that there is only residual staining in head regions. The IAB5 enhancer continues to direct strong expression in the presumptive abdomen. $(E)$ lacZ expression pattern directed by a fusion promoter containing the IAB 5 enhancer and the $2 \mathrm{xPE}$ mesoderm-specific enhancer. The two enhancers are separated by the $\mu$ MAR spacer. An additive pattern is observed consisting of a band of expression in the abdomen (IAB5) as well as ventral staining in the presumptive mesoderm $(2 \mathrm{xPE})$. ( $F)$ Same as $E$ except that the spacer sequence was replaced with Fab- 7 . The distal $2 \mathrm{xPE}$ enhancer is blocked, so that expression is lost in ventral regions. In contrast, IAB5 continues to direct strong expression in the presumptive abdomen.

tenuates, but does not abolish, interactions of the distal IAB5 enhancer (Fig. 2B), but results in a more complete block of the head stripe enhancer (Fig. 2D), as well as a second heterologous enhancer (2XPE; Jiang et al. 1991), which mediates expression in the presumptive mesoderm (cf. Fig. 2F and E).

\section{Fab-7 can block the rhomboid NEE}

Additional fusion promoters were examined to determine the efficacy of Fab-7 attenuator activity. Recent studies suggest that the 330-bp rhomboid (rho) neuroectoderm enhancer, NEE (Ip et al. 1992), is particularly resistant to blocking by the gypsy insulator ( $\mathrm{S}$. Barolo and $M$. Levine, in prep.; H. Cai and $M$. Levine, in prep.). A fusion gene that contains the 500-bp even-skipped (eve) stripe 3 enhancer (Small et al. 1996; Yan et al. 1996) and the rho NEE directs an additive staining pattern, including a central segmentation stripe and two lateral neuroectodermal stripes (Fig. 3A,C). Replacing the spacer DNA with two tandem copies of the 1.2-kb Fab-7 element results in the attenuation of NEE-mediated lateral stripes when monitoring the expression of the leftward white gene (cf. Fig. 3B and A). In contrast, the rightward lacZ promoter shows a selective block in stripe 3 expression (cf. Fig. 3D and C). A single copy of Fab-7 results in a similar, but less complete, block in enhancer-promoter interactions (data not shown). These results suggest that Fab-7 is somewhat less effective than the 340-bp gypsy insulator in blocking the NEE /Cai and Levine 1995; H. Cai and M. Levine, in prep.). In this regard, two copies of Fab-7 appear comparable to one copy of gypsy.

\section{Fab-7 works when positioned far from distal enhancers or target promoters}

Fab-7 is located far from the $A b d-B$ promoter, $>35 \mathrm{~kb}$ downstream of the transcription start site (see Fig. 1 summary). Additional assays were conducted to determine whether Fab-7 could function when placed at a distance from the white and $l a c Z$ test promoters. As shown previously, the rho NEE mediates lateral stripes of gene expression when placed downstream of $1 a c Z,>5$ $\mathrm{kb}$ from the transcription start site (Fig. 3E; Gray and Levine 1996). Fab-7 blocks these lateral stripes when placed between the NEE and $3^{\prime}$ end of lacZ /cf. Fig. 3F and $\mathrm{E})$. The $5^{\prime} \mathrm{H} 1$ enhancer is unaffected, again suggesting that Fab-7 selectively blocks distal, not proximal, enhancers.

In an effort to assess Fab-7 function in the context of a more authentic arrangement of regulatory elements (see Fig. 1 summary|, we positioned Fab-7 and IAB5 far from the white and $l a c Z$ test promoters. The IAB5 enhancer mediates efficient abdominal expression from both promoters when placed downstream of lacZ (Fig. 4A, C, and E). Fab-7 blocks these interactions when inserted between the IAB5 enhancer and 3' end of lacZ (cf. Fig. 4B and $D$ with $A$ and $C$, respectively). This block is comparable to that observed when both Fab-7 and IAB5 are located 5' of lacZ (see Fig. 2B). These results suggest that Fab-7 works equally well in blocking distal enhancers 
Figure 3. Fab-7 attenuates heterologous enhancers. Transgenic embryos were stained and oriented as described in Fig. 2. (A) white expression pattern directed by a fusion promoter that contains the 500-bp eve stripe 3 enhancer and a 330-bp rhomboid (rho) lateral stripe enhancer (NEE). The two enhancers are separated by a $2.5-\mathrm{kb}$ DNA fragment derived from the $\lambda$ bacteriophage. This spacer sequence does not affect either enhancer, so that white is expressed in an additive pattern consisting of stripe 3 and lateral stripes. $(B)$ Same as $A$ except that the $\lambda$ spacer sequence was replaced by two tandem copies of the 1.2-kb Fab-7 element. The rho NEE is selectively attenuated, so that only residual lateral stripes are observed. In con-
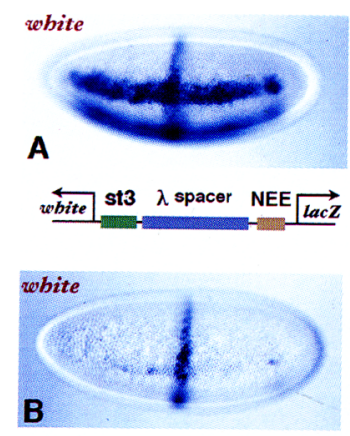

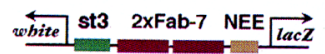

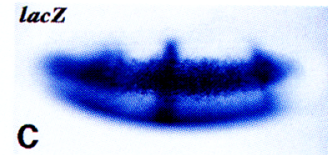

C
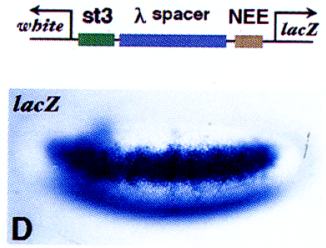

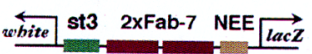
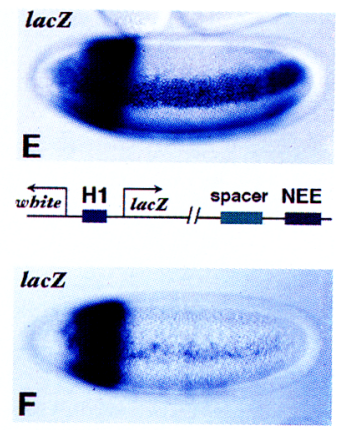

$\stackrel{\text { wite }}{\mathrm{H} 1} \underset{\text { lac Z }}{\longrightarrow}$ Fab-7 NEE trast, the proximal stripe 3 enhancer is unaffected. $(C)$ lac $Z$ staining pattern directed by the same fusion gene as in $A$. The $\lambda$ spacer does not affect either the distal stripe 3 enhancer or the proximal NEE, so that an additive staining pattern is observed. $(D)$ Same as $C$ except that the $\lambda$ spacer was replaced with two copies of Fab-7. The distal stripe 3 enhancer is attenuated, but the proximal NEE is unaffected so that strong lateral stripes are observed. $(E)$ lacZ staining pattern directed by the 200-bp hairy H1 enhancer and the rho NEE. The two enhancers were placed at opposite ends of the lac $Z$ transcription unit, with $\mathrm{Hl} 5^{\prime}$ of lac $Z$ and the NEE downstream of the gene. The 1-kb mouse $\mu$ MAR sequence was inserted between the $3^{\prime}$ end of $l a c Z$ and the distal NEE. An additive staining pattern is observed, consisting of a head stripe (HI) and lateral stripes (NEE). $(F)$ Same as $E$ except that the $3^{\prime}$ spacer sequence was replaced with one copy of the Fab-7 element. The distal NEE is attenuated, so that the lateral stripes are weak. In contrast, the $5^{\prime}-\mathrm{Hl}$ enhancer is unaffected and continues to direct an intense head stripe.

when located either close to or far from the target promoter. Additional assays suggest that Fab-7 also mediates efficient attenuator activity when located far from the distal enhancer. For example, Fab-7 blocks IAB5white interactions when located between the white and lac $Z$ test promoters (cf. Fig. $4 \mathrm{~F}$ and E).

\section{Discussion}

We have presented evidence that Fab- 7 functions as an attenuator that selectively impedes the interaction of distal enhancers with target promoters. These results raise the possibility that specialized DNA elements, such as attenuators, may be normal constituents of complex genetic loci. It has been proposed that Fab-7 functions as a boundary element or insulator, which separates $i a b-6$ and $i a b-7$ so that $i a b-6$ enhancers are not ectopically activated in PS 12 and iab-7 enhancers are not activated in PS 11 (Galloni et al. 1993; Vazquez et al. 1993; Karch et al. 1994). Our studies do not exclude this model, but suggest that an additional function of Fab-7 is the quantitative modulation of IAB5- $A b d-B$ interactions. This ensures that only low levels of $A b d-B$ are expressed in anterior regions (PS 10) of the presumptive abdomen.

Figure 4. The Fab-7 insulator works when far from either the target promoter or distal enhancer. Transgenic embryos were stained and oriented as described in Figure 2. $(A)$ white expression driven by the hairy $\mathrm{H} 1$ and IAB 5 enhancers. The $\mathrm{H} 1$ enhancer is located $5^{\prime}$ of both the white and lac $Z$ transcription units whereas IAB5 is positioned downstream of lacZ. The $\mu$ MAR spacer sequence was placed between the $3^{\prime}$ end of $l a c Z$ and the distal IAB5 enhancer. An additive white expression pattern is observed, consisting of a head stripe $(\mathrm{H} 1)$ and a band of staining in the presumptive abdomen. $(B)$ Same as $A$ except that the $3^{\prime}$ spacer sequence was re-
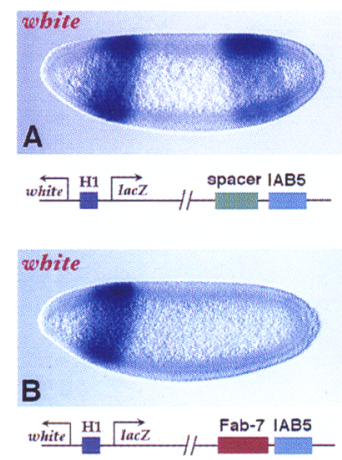
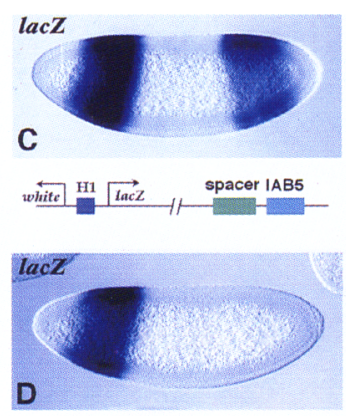

white $\mathrm{H}_{\text {1 }}^{\text {lacZ }}$
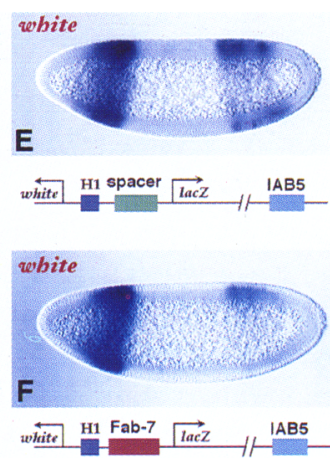

placed with the Fab-7 element. Expression from the IAB5 enhancer is blocked, but the $5^{\prime}-\mathrm{H} 1$ enhancer is unaffected so that white continues to be expressed in the limits of a head stripe. $|C|$ lac $Z$ expression driven by the $\mathrm{H} 1$ and IAB 5 enhancers. This is the same fusion gene as in $A$, except that the rightward lac $Z$ gene is being monitored. Strong staining is observed in both anterior (H1) and posterior (IAB5) regions. $(D)$ Same as $C$ except that the spacer was replaced with Fab-7. The distal IAB5 enhancer is blocked, so that expression is directed solely by the $5^{\prime} \mathrm{Hl}$ enhancer. $(E)$ white expression pattern directed by the $\mathrm{Hl}$ and IAB 5 enhancers. The fusion gene being analyzed is a variant of that shown in $A-D$. The $H 1$ enhancer is $5^{\prime}$, the IAB5 enhancer is $3^{\prime}$ of lac $Z$, but the spacer sequence is positioned between the $5^{\prime}-\mathrm{H} 1$ enhancer and the lacZ transcription start site. An additive white expression pattern is observed consisting of the anterior head stripe $(\mathrm{HI})$ and expression in the presumptive abdomen (IAB5). (F) Same as $E$ except that the 5' spacer sequence was replaced with Fab-7. The distant IAB5 enhancer (now located $\sim 5 \mathrm{~kb}$ from Fab-7) is selectively attenuated, so that only residual staining is observed in the abdomen. In contrast, the proximal $\mathrm{Hl}$ enhancer continues to direct white expression within the limits of a head stripe. 


\section{Mechanism of Fab-7 activity}

Fab-7 may function in a manner analogous to insulator DNAs, including $s c s, s c s^{\prime}$, and the $s u(H w)$ insulator contained within the gypsy retrotransposon (Kellum and Schedl 1991, 1992; Geyer and Corces 1992; Dorsett 1993|. scs and scs' flank the hsp70 locus and are thought to prevent the heat induction of neighboring genes (Kellum and Schedl 1991). Previous transgenic expression assays have shown that these insulators can also block the interactions of distal enhancers with a target promoter in the context of synthetic gene complexes (Cai and Levine 1995; Scott and Geyer 1995).

At least two models can be envisioned for the Fab-7 attenuator, as summarized in Figure 5. We favor the notion that Fab-7 functions as an autonomous cis regulatory element that blocks the looping and/or scanning of distal enhancers to the target promoter (Fig. 5A). Perhaps Fab-7 mimics the core promoter by containing sites for looping proteins, which are required for long-range interactions of a distal enhancer with the promoter. Another way that Fab-7 might mimic the core promoter is by bending DNA. Fab-7 binding proteins might induce structural changes similar to those mediated by the TFIID complex on the core promoter (Oelgeschlager et al. 1996).

A second type of model invokes the organization of chromatin into separate nuclear domains or loops. For example, Fab-7 may work in conjunction with other boundary elements in the BX-C to organize $i a b-6$ and iab-7 into separate domains (Fig. 5B; Vazquez et al. 1993). This would prevent enhancer hitchhiking, whereby interactions between $i a b-6$ enhancers and the $A b d-B$ promoter would cause fortuitous interactions be-

A
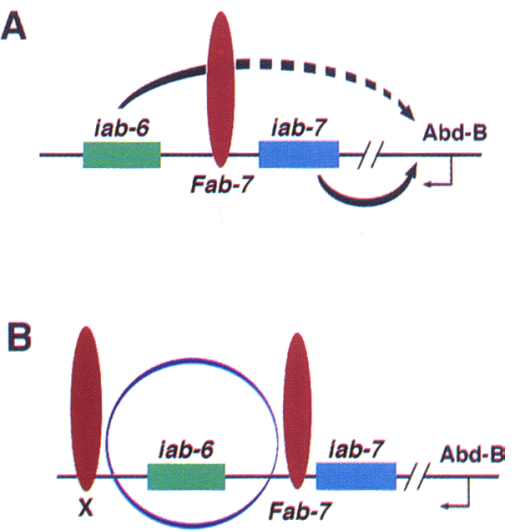

Figure 5. Models for Fab-7 function. $(A)$ We propose that Fab-7 functions as an autonomous cis regulatory element that selectively blocks or impedes the interactions of distal enhancers with a target promoter. The mechanism for this directional repression is unknown, but it is possible that Fab-7 might bind looping proteins required for the interaction of distal enhancers with a target promoter. $(B)$ It has been suggested that Fab-7 interacts with other boundary elements $(\mathrm{X})$ to form a chromatin loop domain that separates the $i a b-6$ cis regulatory region from iab-7. tween $i a b-7$ enhancers and the promoter. In addition, separate loop domains would block cross-enhancer interactions whereby activators bound to $i a b-6$, such as those encoded by the trithorax group, would interfere with Pc-G repressors in $i a b-7$, resulting in the ectopic activation of $i a b-7$ enhancers in PS 11 (Vasquez et al. 1993). It is conceivable that Fab-7 associates with endogenous insulators flanking the integrated transgene to form loop domains (Corces 1995). The two models depicted in Figure 5 are not mutually exclusive in that the formation of loop domains might induce structural changes in Fab-7, similar to those detected at the core promoter (Oelgeschlager et al. 1996).

\section{Multiple modes of Fab-7 regulation}

Fab-7 plays an essential role in restricting $A b d-B$ expression to posterior parasegments (Busturia and Bienz 1993; Galloni et al. 1993). It is conceivable that the full-length Fab-7 element functions as both an attenuator and PRE. As discussed earlier, a 3.7-kb DNA fragment that encompasses the core Fab-7 element was shown to repress a $b x d-U b x$ fusion promoter in a Pc-G-dependent fashion (Busturia and Bienz 1993). This fragment was also found to silence the expression of a mini-white gene and bind Pc proteins on polytene chromosomes (Zink and Paro 1995|. Fab-7 mutations might disrupt a PRE that normally attenuates $A b d-B$ expression in PS 11 and other anterior parasegments.

We cannot exclude the possibility that the core Fab-7 element resides near a PRE. However, several observations suggest that attenuator activity does not depend on Pc-G proteins. First, we have demonstrated that Fab-7 works as an attenuator in precellular embryos, which is well before the time when PREs silence the expression of homeotic selector genes in anterior parasegments /this usually occurs after germ-band elongation; Bienz and Muller 1995; Paro 1995|. Second, Fab-7 activity is not affected by mutations in $P c-G$ genes, including $P c$ and the maternally expressed extra sex combs (esc) gene (J. Zhou, unpubl.). Finally, a well-characterized PRE, the $1.6-\mathrm{kb}$ fragment from the $p b x$ region of the $U b x$ domain (Zhang and Bienz 1992; Chan et al. 1994), does not function as an attenuator when placed between the $\mathrm{Hl}$ and IAB5 enhancers (P. Szymanski, unpubl.). These results indicate that attenuation and PRE-mediated silencing represent separate functions.

The demonstration that Fab-7 functions as an attenuator suggests that it can impede interactions between distal enhancers, such as IAB5, and the $A b d-B$ promoter. In principle, IAB5 has the capacity to direct strong expression in PS 10 , where $A b d-B$ is normally expressed at low levels (Busturia and Bienz 1993). Fab-7 might be required to ensure weak expression in this region. It has been proposed that there may be another boundary element located between $i a b-5$ and $i a b-6$ (Vasquez et al. 1993). Redundancy of Fab-7 and a second attenuator could explain why Fab-7 mutants do not exhibit obvious homeotic transformations of PS 10 (A5) tissues (Gyurkovics et al. 1990; Galloni et al. 1993). 
We propose that specialized DNAs such as Fab-7 might be relatively common constituents of complex genetic loci. Metazoan promoter regions can be modular, and contain a series of nonoverlapping enhancers (e.g., Goto et al. 1989; Harding et al. 1989; Howard and Struhl 1990; Riddihough and Ish-Horowicz 1991). These enhancers may be interlaced with specialized DNA elements. In the simplest view, enhancers integrate positive and negative regulatory information to determine the spatial limits of gene expression. Specialized DNAs, such as attenuators, might control the levels of expression by quantitatively modulating enhancer-promoter interactions.

\section{Materials and methods \\ P-element transformation and in situ hybridization}

P-element transformation vectors containing $\operatorname{lac} Z$ and white reporter genes were introduced into the Drosophila germline by injection of yw67 embryos as described in Small et al. (1992). In situ hybridizations were performed as described (Tautz and Pfeifle 1989; Jiang et al. 1991), using digoxigenin-UTP-labeled antisense RNA to lacZ or white. Multiple transgenic lines for each construct were generated, and four to six independent lines were independently stained.

\section{Construction of lacZ reporter genes}

The 1.2-kb Fab-7 DNA fragment was obtained by PCR amplification from Drosophila genomic DNA. BamHI restriction sites were introduced into the primers. A final BamHI fragment was recovered that spans nucleotide positions 84,594 to 83,423 within the complete BX-C sequence (Lewis et al. 1995; Martin et al. 1995). A 1-kb MAR spacer sequence was also obtained by PCR amplification using primers homologous to the 3 ' matrix attachment region near the $3^{\prime}$ intronic enhancer of the $\operatorname{Ig} \mu$ chain gene (Forrester et al. 1994). The 2.5-kb $\lambda$ spacer was obtained by digesting total $\lambda$ genomic DNA with BstYI.

The fusion genes shown in Figures $2 A-D$ were prepared by inserting a 200-bp SpeI-BamHI fragment from the hairy stripe 1 enhancer (kindly provided by G. Struhl, Columbia University, New York, NY) into the C4PLZ vector (see Cai and Levine 1995). A l-kb SalI DNA fragment from the IAB5 enhancer (Busturia and Bienz 1993) was then inserted in the SphI site, located between the hairy stripe 1 enhancer and $l a c Z$ transcription unit. The 1-kb spacer or the $1.2-\mathrm{kb}$ Fab-7 element was subsequently cloned in the unique BamHI site between the hairy stripe 1 and IAB5 enhancers.

The fusion genes shown in Figures $2 \mathrm{E}$ and $\mathrm{F}$ were prepared by replacing the stripe 1 enhancer with a 500-bp NotI-BamHI fragment containing two copies of the proximal enhancer from the twist promoter region (2XPE; see Jiang et al. 1991).

The eve stripe 3-rho NEE fusion gene shown in Figures 3A-D was prepared by inserting a 500-bp eve stripe 3 enhancer (Small et al. 1993) into the XbaI site of the pBluescript II SK plasmid vector (Stratagene). Subsequently, a 330-bp StyI-XhoI DNA fragment containing the NEE rho enhancer (Ip et al. 1992) was cloned into the EcoRI site. The enhancers were excised as a NotI-EcoRV fragment and ligated into the EcoRI site of the -42 pCaSpeR vector (Small et al. 1992). Either one or two tandem copies of the Fab-7 element was inserted in the BamHI site located between the eve stripe 3 and NEE enhancers.

The fusion genes shown in Figures $3 \mathrm{E}$ and $\mathrm{F}$ were prepared by inserting the 200-bp SpeI-BamHI hairy stripe 1 enhancer into the C4PLZ vector. A 700-bp (full-length) rho NEE was inserted into a StuI site at the $3^{\prime}$ of the lac $Z$ transcription unit. The MAR spacer sequence or the Fab-7 fragment was inserted in a unique $B g l$ II site located between the distal NEE and $3^{\prime}$ end of lacZ.

The fusion genes shown in Figures 4A-D were prepared by inserting the 1-kb IAB5 enhancer in the 3' StuI site in place of the rho NEE. Finally, those shown in Figures $4 \mathrm{E}$ and $\mathrm{F}$ were prepared by inserting either the spacer or Fab-7 fragment into the unique BamHI site located $5^{\prime}$ of the white and lacZ transcription units.

\section{Acknowledgments}

We thank Mariann Bienz for providing IAB5 Fab-7 DNA, and Gary Struhl for his fine unpublished work on the hairy stripe 1 enhancer. We thank David Arnosti, Bill McGinnis, and Kees Murre for critically reading the manuscript. We also thank Liezelle de la Pena for technical assistance, and Christian Burks for help with computational analyses. This work was funded by a grant from the National Institues of Health (GM34431) and done in part under the auspices of the Department of Energy with funds from the UC/LANL CULAR program (UCDRD \#9624).

The publication costs of this article were defrayed in part by payment of page charges. This article must therefore be hereby marked "advertisement" in accordance with 18 USC section 1734 solely to indicate this fact.

\section{Note added in proof}

Similar results have been obtained by Hagstrom et al. (this issue).

\section{References}

Bienz, M. and J. Muller. 1995. Transcriptional silencing of homeotic genes in Drosophila. BioEssays 17: 775-784.

Boulet, A.M., A. Lloyd, and S. Sakonju. 1991. Molecular definition of the morphogenetic and regulatory functions of the cis-regulatory elements of the Drosophila $A b d-B$ homeotic gene. Development 111: 393-405.

Busturia, A. and M. Bienz. 1993. Silencers in Abdominal-B, a homeotic Drosophila gene. EMBO I. 12: 1415-1425.

Cai, H. and M. Levine. 1995. Modulation of enhancer-promoter interactions by insulators in the Drosophila embryo. Nature 376: 533-536.

Casanova, J., E. Sanchez-Herrero, and G. Morata. 1986. Identification and characterization of a parasegment specific regulatory element of the Abdominal-B gene of Drosophila. Cell 47: 627-635.

Celniker, S.E., D.J. Keelan, and E.B. Lewis. 1989. The molecular genetics of the bithorax complex of Drosophila-Characterization of the Abdominal-B domain. Genes \& Dev. 3: 14241436.

Celniker, S.E., S. Sharma, D. Keelan, and E.B. Lewis. 1990. The molecular genetics of the bithorax complex of Drosophila: cis-regulation in the Abdominal-B domain. EMBO $I$. 9: 4277-4285.

Chan, C.S., L. Rastelli, and V. Pirrotta. 1994. A Polycomb response element in the $U b x$ gene that determines an epigenetically inherited state of repression. EMBO J. 13: 25532564.

Chiang, A., M.B. O'Connor, R. Paro, J. Simon, and W. Bender. 1995. Discrete Polycomb-binding sites in each parasegmental domain of the bithorax complex. Development 121: 1681-1689.

Corces, V.G. 1995. Chromatin insulators: Keeping enhancers 
under control. Nature 376: 462-463.

DeLorenzi, M. and M. Bienz. 1990. Expression of Abdominal-B homeoproteins in Drosophila embryos. Development 108: 323-329.

Dorsett, D. 1993. Distance-independent inactivation of an enhancer by the suppressor of Hairy-wing DNA-binding protein of Drosophila. Genetics 134: 1135-1144.

Forrester, W.C., C. van Genderen, T. Jenuwein, and R. Grosschedl. 1994. Dependence of enhancer-mediated transcription of the immunoglobulin $\mu$ gene on nuclear matrix attachment regions. Science 265: 1221-1225.

Galloni, M., H. Gyurkovics, P. Schedl, and F. Karch. 1993. The bluetail transposon: Evidence for independent cis-regulatory domains and domain boundaries in the bithorax complex. EMBO I. 12: 1087-1095.

Geyer, P.K. and V.G. Corces. 1992. DNA position-specific repression of transcription by a Drosophila zinc finger protein. Genes \& Dev. 6: 1865-1873.

Goto, T., P.M. Macdonald, and T. Maniatis. 1989. Early and late periodic patterns of even-skipped expression are controlled by distinct regulatory elements that respond to different spatial cues. Cell 57: 413-422.

Gray, S. and M. Levine. 1996. Short-range transcriptional repressors mediate both quenching and direct repression within complex loci in Drosophila. Genes \& Dev. 10: 700710.

Gyurkovics, H., J. Gausz, J. Kummer, and F. Karch. 1990. A new homeotic mutation in the Drosophila bithorax complex removes a boundary separating two domains of regulation. EMBO J. 9: 2579-2585.

Hagstrom, K., M. Muller, and P. Schedl. 1996. Fab-7 functions as a chromatin domain boundary to ensure proper segment specification by the Drosophila bithorax complex. Genes \& Dev. (this issue).

Harding, K. and M. Levine. 1988. Gap genes define the limits of Antennapedia and Bithorax gene expression during early development in Drosophila. EMBO J. 7: 205-214.

Harding, K., T. Hoey, R. Warrior, and M. Levine. 1989. Autoregulatory and gap gene response elements of the evenskipped promoter of Drosophila. EMBO I. 8: 1205-1212.

Howard, K.R. and G. Struhl. 1990. Decoding postitional information-regulation of the pair-rule gene hairy. Development 110: 1223-1231.

Ip, Y.T., R.E. Park, D. Kosman, E. Bier, and M. Levine. 1992. The dorsal gradient morphogen regulates stripes of rhomboid expression in the presumptive neuroectoderm of the Drosophila embryo. Genes \& Dev, 6: 1728-1739.

Jiang, J., D. Kosman, Y.T. Ip, and M. Levine. 1991. The dorsal morphogen gradient regulates the mesoderm determinant twist in early Drosophila embryos. Genes \& Dev. 5: 18811891

Karch, F., B. Weiffenbach, M. Peifer, W. Bender, I. Duncan, S. Celniker, M. Crosby, and E.B. Lewis. 1985. The abdominal region of the bithorax complex. Cell 43: 81-90.

Karch, F., M. Galloni, L. Sipos, J. Gausz, H. Gyurkovics, and P. Schedl. 1994. Mcp and Fab-7: Molecular analysis of putative boundaries of cis-regulatory domains in the bithorax complex of Drosophila melanogaster. Nucleic Acids Res. 22: $3138-3146$.

Kellum, B. and P. Schedl. 1991. A position effect assay for boundaries of higher order domains. Cell 64: 941-950.

- 1992. A group of scs elements function as domain boundaries in an enhancer-blocking assay. Mol. Cell. Biol. 12: $2424-2433$.

Kuziora, M.A. and W. McGinnis. 1988. Autoregulation of a Drosophila homeotic selector gene. Cell 55: 477-485.
Lewis, E.B. 1978. A gene complex controlling segmentation in Drosophila. Nature 276: 565-570.

Lewis, E.B., I.D. Knafels, D.R. Mathog, and S.E. Celniker. 1995. Sequence analysis of the cis-regulatory regions of the bithorax complex of Drosophila. Proc. Natl. Acad. Sci. 92: 84038407.

Martin, C.H., C.A. Mayeda, C.A. Davis, C.L. Ericsson, J.D. Knafels, D.R. Mathog, S.E. Celniker, E.B. Lewis, and M.J. Palazzolo. 1995. Complete sequence of the bithorax complex of Drosophila. Proc. Natl. Acad. Sci. 92: 8398-8402.

Oelgeschlager, T., C.M. Chiang, and R.G. Roeder. 1996. Topology and reorganization of human TFIID-promoter complex. Nature 382: 735-738.

Orlando, V. and R. Paro. 1993. Mapping Polycomb-repressed domains in the bithorax complex using in vivo formaldehyde cross-linked chromatin. Cell 75: 1187-1198.

Paro, R. 1995. Propagating memory of transcriptional states. Trends Genet. 11: 295-297.

Riddihough, G. and D. Ish-Horowicz. 1991. Individual stripe regulatory elements in the Drosophila hairy promoter respond to maternal, gap, and pair-rule genes. Genes \& Dev. 5: 840-854.

Sanchez-Herrero, E. 1991. Control of the expression of the bithorax complex abdominal-A and Abdominal-B by cisregulatory regions in Drosophila embryos. Development 111: 437-448.

Sanchez-Herrero, E., I. Vernos, R. Marco, and G. Morata. 1985. Genetic organization of the Drosophila bithorax complex. Nature 313: 108-113.

Scott, K.S. and P.K. Geyer. 1995. Effects of the $s u(H W)$ insulator protein on the expression of the divergently transcribed Drosophila yolk protein genes. EMBO I. 14: 6258-6267.

Small, S., A. Blair, and M. Levine. 1992. Regulation of evenskipped stripe 2 in the Drosophila embryo. EMBO $/$. 11: 4047-4057.

Small, S., D.N. Arnosti, and M. Levine. 1993. Spacing ensures autonomous expression of different stripe enhancers in the even-skipped promoter. Development 119: 767-772.

Small, S., A. Blair, and M. Levine. 1996. Regulation of two pairrule stripes by a single enhancer in the Drosophila embryo. Dev. Biol. 175: 314-324.

Tautz, D. and C. Pfeifle. 1989. A non-radioactive in situ hybridization method for the localization of specific RNAs in Drosophila embryos reveals translational control of the segmentation gene hunchback. Chromosoma 98: 81-85.

Tiong, S.Y.K., L.M. Bone, and J.R.S. Whittle. 1985. Recessive lethal mutations within the bithorax complex in Drosophila. Mol. Gen. Genet. 200: 335-344.

Vazquez, J., G. Farkas, M. Gaszner, A. Udvardy, M. Muller, K. Hagstrom, H. Gyurkovics, L. Sipos, J. Gausz, M. Galloni, I. Hogga, F. Karch, and P. Schedl. 1993. Genetic and molecular analysis of chromatin domains. Cold Spring Harbor Symp. Quant. Biol. 58: 45-54.

Wedeen, C., K. Harding, and M. Levine. 1986. Spatial regulation of Antennapedia and bithorax gene expression by the Polycomb locus in Drosophila. Cell 44: 739-748.

Yan, R., S. Small, C. Desplan, C. Dearolf, and J.E. Darnell, Jr. 1996. Identification of a STAT gene that functions in Drosophila development. Cell 84: 421-430.

Zhang, C.C. and M. Bienz. 1992. Segmental determination in Drosophila conferred by hunchback $(h b)$, a repressor of the homeotic gene Ultrabithorax $(U b x)$. Proc. Natl. Acad. Sci. 89: 7511-7515.

Zink, D. and R. Paro. 1995. Drosophila Polycomb-group regulated chromatin inhibits the accessibility of a trans-activator to its target DNA. EMBO I. 14: 5660-5671. 


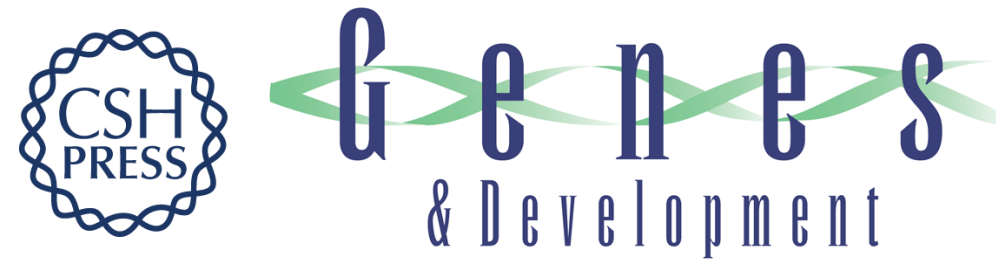

\section{The Fab-7 element of the bithorax complex attenuates enhancer-promoter interactions in the Drosophila embryo.}

J Zhou, S Barolo, P Szymanski, et al.

Genes Dev. 1996, 10:

Access the most recent version at doi:10.1101/gad.10.24.3195

References This article cites 48 articles, 19 of which can be accessed free at:

http://genesdev.cshlp.org/content/10/24/3195.full.html\#ref-list-1

License

Email Alerting

Service

Receive free email alerts when new articles cite this article - sign up in the box at the top right corner of the article or click here.

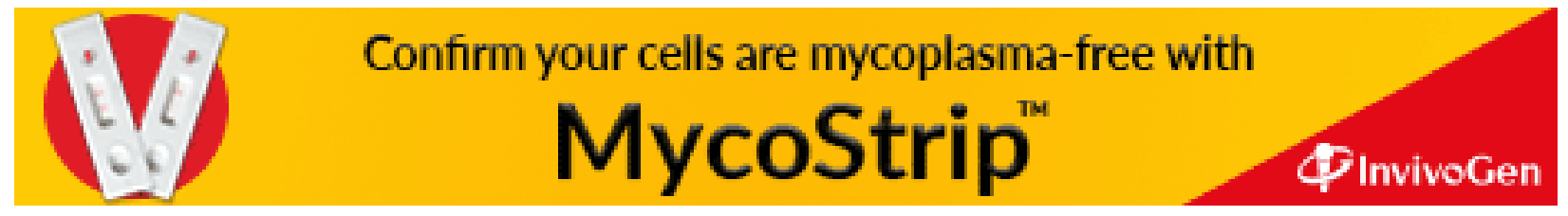

